\title{
An Information Flow Filter Approach to Cooperative Vehicle Control
}

\author{
Ulf Pilz* Andrey P. Popov* Herbert Werner* \\ * Institute of Control Systems, Hamburg University of Technology \\ D-21073 Hamburg, Germany \\ e-mail: \{ulf.pilz, andrey.popov, h.werner $\}$ tu-harburg.de.
}

\begin{abstract}
This paper extends an previously proposed information flow filter approach to cooperative vehicle control to guarantee stability of a vehicle formation under arbitrary communication topologies. A systematic design technique for this information flow filter is the main contribution of this work. For this purpose an alternative interpretation of a separation theorem that allows the independent design of the information flow filter and the controllers for the vehicles is presented. The advantages of this approach are illustrated in simulation studies.
\end{abstract}

Keywords: Cooperative control, multi-agent systems, robust stability, distributed control, autonomous systems

\section{INTRODUCTION}

The field of cooperative control of vehicles is characterized by the condition that information is shared between the vehicles since they are not physically connected. Research activities in this field increased over the last several years due to its broad variety of potential applications like unmanned aerial vehicles [Bouabdallah and Siegwart, 2007], mobile robotics [Ren, 2010a,b], autonomous underwater vehicles [Kalantar and Zimmer, 2007] and space-based interferometers [Unwin et al., 2008]. Common threads in these different fields include formation control [Fax and Murray, 2004; Caughman et al., 2005], attitude alignment [Bauso et al., 2003; Ren, 2007], flocking [Olfati-Saber, 2006; Tanner et al., 2007], coverage control [Cortés et al., 2004; Hussein and Stipanović, 2006], rendezvous strategies [Lin et al., 2003; Cortés et al., 2006] and task and role assignment [Klavins and Murray, 2003].

The cooperative control framework considered in this paper has been proposed in [Fax and Murray, 2004]. In this framework vehicles obtain information about the outputs of other vehicles in the formation either by sensing or by communicating with each other. Furthermore, the authors of [Fax and Murray, 2004] also introduced an information flow filter scheme that guarantees formation stability for arbitrary communication topologies. A separation principle that allows for the design of local controllers for the vehicles and information flow filters independently has also been established in that work.

Designing local controllers for a group of autonomous vehicles in a formation has also been studied in [Borrelli and Keviczky, 2006; Gu, 2008] where state feedback controllers are synthesized to stabilize a formation of identical vehicles. Output-feedback control of passive systems has been considered in [Chopra and Spong, 2006], whereas in [Massioni and Verhaegen, 2009] a decomposition approach has been proposed. A different method is proposed in
[Wang and Elia, 2009] where a robust control approach is applied.

The main contribution of this paper is an alternative interpretation of a separation principle that allows for a systematic design of the information flow filter using mixed sensitivity tuning rules and standard tools from $\mu$ synthesis. Compared with the results given in $[\mathrm{Gu}, 2008$; Wang and Elia, 2009], where the number of agents is only allowed to vary between known limits, the proposed approach can handle an arbitrary number of agents in the formation. The consideration of directed communication topologies is an extension compared the design method in [Borrelli and Keviczky, 2006; Chopra and Spong, 2006; Gu, 2008; Liu and Jia, 2010]. Furthermore, unknown communication topologies are addressed in the filter synthesis method. For this purpose the communication topology is modeled as a complex parametric uncertainty and handled in a robust control framework.

This paper is organized as follows. In Section 2 the cooperative control framework and some graph theory is reviewed. Section 3 presents the main contribution of this paper: based on an alternative interpretation of a separation theorem, we derive a synthesis method for the information flow filter that guarantees robust stability for arbitrary topologies, and allows to tune performance in a $\mathcal{H}_{\infty}$ loop-shaping sense. Section 4 illustrates the proposed information flow synthesis method and compares it with previous techniques. Finally, in Section 5 conclusions are drawn.

\section{PRELIMINARIES}

\subsection{Notation}

$\mathbb{R}_{+}$is the set of all non-negative real numbers and $\mathbb{N}_{+}$is the set of non-negative integers; $\mathrm{j}$ is the imaginary unit; $I_{q}$ denotes the $q \times q$ identity matrix; $A^{\top}$ denotes the transpose of a matrix $A$. $\mathbb{R}^{p \times q}, \mathbb{C}^{p \times q}$ are the sets of $p \times q$ real 
and complex matrices correspondingly, $\otimes$ is the Kronecker product. Furthermore, $Q_{(m)}$ is a shorthand notation for $Q \otimes I_{m}$ and $\widehat{Q}=I_{N} \otimes Q$, where $Q$ may represent either a matrix or a dynamic system. Finally, $\mathcal{F}_{L}(P(z), K(z))$ denotes the lower linear fractional transformation (LFT) of $P(z)$ with $K(z)$ [Zhou et al., 1996].

\subsection{Graph Theory}

Concepts from graph theory are commonly used to model communication and sensing topologies in a multi-agent system (MAS). First, we recall a few basic definitions (see, e.g., [Diestel, 2005]).

A graph $\mathcal{G}$ consists of a vertex set $\mathcal{V}(\mathcal{G})$ and an edge set $\mathcal{E}(\mathcal{G})$, where $\mathcal{V}(\mathcal{G})=\left\{v_{1}, \ldots, v_{n}\right\}$ and $\mathcal{E}(\mathcal{G}) \subseteq \mathcal{V} \times \mathcal{V}$. For a directed graph $\mathcal{E}(\mathcal{G}) \subset\left\{e_{i j}=\left(v_{j}, v_{i}\right): v_{j}, v_{i} \in \mathcal{V}(\mathcal{G})\right\}$. We assume that there are no self-loops, i.e., $(i, i) \notin \mathcal{E}, i=$ $1, \ldots, N$, where $N$ denotes the number of vertices. For undirected graphs the equation $e_{i j}=e_{j i}$ holds. If $\left(v_{j}, v_{i}\right)$ is an edge of $\mathcal{G}$, then we say that $v_{j}$ is a neighbor of $v_{i}$. The set of neighbors of vertex $v_{i}$ is defined as $\mathcal{N}_{i}\left(\mathcal{G}, v_{i}\right)=\left\{v_{j}:\left(v_{j}, v_{i}\right) \in \mathcal{E}(\mathcal{G})\right\}$. The adjacency matrix $\mathcal{A}=\left[a_{i j}\right] \in \mathbb{R}^{n \times n}$ associated with $\mathcal{G}$ is defined such that $a_{i j}$ has a positive value if $\left(v_{j}, v_{i}\right) \in \mathcal{E}$ and $a_{i j}=0$ otherwise. The normalized Laplacian matrix $\mathcal{L}=\left[l_{i j}\right]$ associated with $\mathcal{A}$ is defined as

$$
l_{i j}= \begin{cases}1 & \text { if } i=j \text { and }\left|\mathcal{N}_{i}\right| \neq 0 \\ -\frac{1}{\left|\mathcal{N}_{i}\right|} & \text { if } j \in \mathcal{N}_{i}, \\ 0 & \text { otherwise, }\end{cases}
$$

where $\left|\mathcal{N}_{i}\right|$ indicates the cardinality of the set of neighbors $\mathcal{N}_{i}$.

According to the definition in (1) the row-sums of $\mathcal{L}$ are zero. Hence, $\mathcal{L}$ always has a zero eigenvalue, $\lambda_{1}=0$, which corresponds to the eigenvector $\mathbb{1}=(1, \ldots, 1)^{\top}$. It follows from Geršgorin's disk theorem that all eigenvalues of the normalized Laplacian matrix $\mathcal{L}$ are located in a unit disk centered at $1+\mathrm{j} 0$.

\subsection{Formation Modeling and Communication Topology}

We consider a formation control problem for a group of $N$ identical vehicles, each represented by a linear timeinvariant state space model $P(z)$ with $h$ inputs and $q$ outputs. Assuming internal stability of a single vehicle, we are interested in synthesis of a distributed local controller $K(z)$. Fig. 1 shows a diagram of the whole formation.

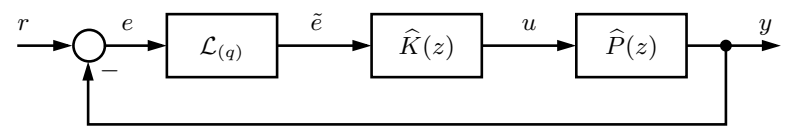

Fig. 1. Feedback loop of the formation

To describe the formation of vehicles and their local controllers in a compact form we make use of Kronecker algebra. In Fig. 1, $u \in \mathbb{R}^{N h}$ are the control inputs for the vehicles, whereas $y, r, e \in \mathbb{R}^{N q}$ are outputs of interest for the formation, reference inputs representing the intended formation and control errors, respectively. $\tilde{e} \in \mathbb{R}^{N q}$ is a measure for the relative error of the vehicles in the formation. The normalized graph Laplacian matrix on formation level $\mathcal{L}_{(q)}=\mathcal{L} \otimes I_{q}$ formally describes the communication structure between the vehicles which is modeled as a directed graph. In this paper we consider graphs which are connected by a directed spanning tree. The nodes of this graph represent the vehicles and the edges indicate a communication link between the vehicles. Note that a vehicle can only receive output signals from vehicles which are members of the neighborhood of this vehicle.

\section{INFORMATION FLOW FRAMEWORK AND INFORMATION FLOW FILTER SYNTHESIS}

In this section it is shown how the information flow filter proposed in [Fax and Murray, 2004] is used to improve stability and performance of a cooperative control scheme by providing every vehicle in the formation with a common reference point. For this purpose a representation of this scheme is introduced that enables a systematic design of an information flow filter using standard tools from robust control theory. A mild assumption which has also been proposed in [Fax and Murray, 2004] is made: namely that the information flow filter is strictly proper to account for at least one time step delay in the transmission of information. The scheme proposed in FFax and Murray, 2004] can be represented as shown in Fig. 2, where $R(z)=$ $\left(I_{q}+F(z)\right)^{-1} F(z)$, and $F(z)$ is an information flow filter. Theorem 1. (Fax and Murray [2004]). Given a filter $R(z)$ the formation dynamics are stabilized if and only if the negative feedback interconnection of $F(z)$ with $\mathcal{L}$ is stable and $K(z)$ stabilizes $P(z)$.

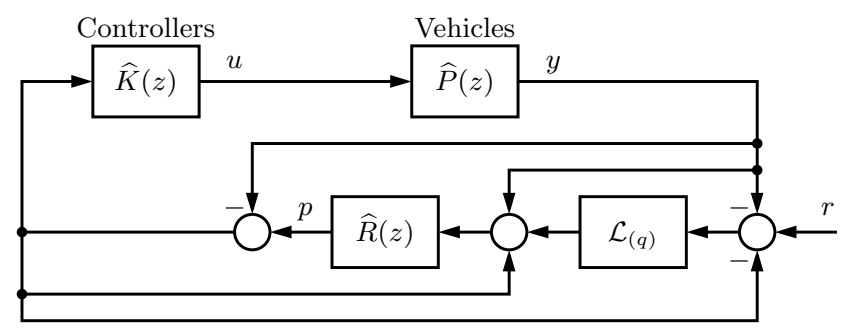

Fig. 2. Information flow filter scheme proposed in [Fax and Murray, 2004]

\subsection{Interpretation of the Separation Principle}

Next we present an alternative representation of the block diagram in Fig. 2. We have

$$
p=\widehat{R}(z) p+\widehat{R}(z) \mathcal{L}_{(q)}(r-p) .
$$

Now substituting for $\widehat{R}(z)=\left(I_{N q}+\widehat{F}(z)\right)^{-1} \widehat{F}(z)$ and multiplying by $\left(\mathcal{L}_{(q)}+\widehat{F}(z)\right)^{-1}$ from left leads to

$$
p=\left(I_{N q}+\widehat{F}(z)\right)^{-1} \widehat{F}(z)\left(p+\mathcal{L}_{(q)} r-\mathcal{L}_{(q)} p\right) .
$$

From there it follows that

$$
p=\left(I_{N q}+\widehat{F}(z) \mathcal{L}_{(q)}\right)^{-1} \widehat{F}(z) \mathcal{L}_{(q)} r=G_{p r}(z) r .
$$

Equation (4) is depicted as the first feedback loop in Fig. 3. The second feedback loop consists of the vehicles and their local controllers. For the second loop one obtains

$$
y=\left(I_{N q}+\widehat{P}(z) \widehat{K}(z)\right)^{-1} \widehat{P}(z) \widehat{K}(z) p=G_{y p}(z) p .
$$


These equations are expressed in a block diagram in Fig. 3, which does not only give a visual representation of the separation principle proved in [Fax and Murray, 2004], but also shows that in fact the only communicated signal in this scheme is the information signal $p$.

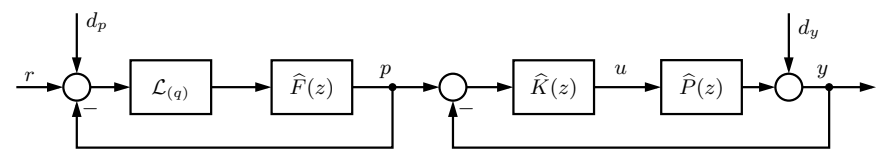

Fig. 3. Graphical interpretation of the separation theorem

The overall transfer function of the system from the reference input $r$ to the output of the agents $y$ is $G_{y r}=$ $G_{y p} G_{p r}$ and the design parameters of the overall system are the dynamical systems $\widehat{F}(z)$ and $\widehat{K}(z)$.

In Fig. 3 two different types of disturbances are considered in the cooperative control framework: disturbances acting on the agents, such as output disturbances $d_{y}$ and disturbances on the communication $d_{p}$. As a result of the decoupling shown above, an output disturbance $d_{y_{i}}$ will act only in the control loop of vehicle $i$ and will not affect the other vehicles. In contrast, a communication disturbance $d_{p_{i}}$ will have an effect on the information of vehicles of which vehicle $i$ is neighbor, as it can be considered as a change in the reference input $r_{i}$. Hence, one can design the local controller $K(z)$ for a good output disturbance rejection, and design $F(z)$ so that information consensus and a good reference tracking are achieved.

\subsection{Information Flow Filter Synthesis}

In [Fax and Murray, 2004] it is assumed that the information flow filter is designed and tuned heuristically, which is realistic only for SISO systems. In this section it is shown how a robust controller synthesis approach can be applied to synthesize information flow filters $\widehat{F}(z)$ that guarantee robust stability of the MAS for an arbitrary number of agents and arbitrary communication topologies, and impose constraints on the performance.

Theorem 2. Given a controller $K(z)$ that stabilizes $P(z)$, and a filter $F(z)$, a MAS will be stable for any number of agents $N$ and for any communication topology if and only if the spectral radius of $R(z)$ satisfies $\rho\left(R\left(e^{\mathrm{j} \omega}\right)\right)<1$, $\forall \omega \in[-\pi, \pi]$.

Proof The stability of the MAS will depend only on the stability of $G_{p r}(z)$ since $K(z)$ is a stabilizing controller by assumption. According to the stability test developed in [Fax and Murray, 2004] the system $G_{p r}(z)$ will be stable if the feedback interconnections of $F(z)$ with the negative eigenvalues $-\lambda_{i}$ of all conceivable topologies $\mathcal{L}$ (shown in Fig. 4a) are stable. For the eigenvalues of $\mathcal{L}$ one can write $\lambda_{i}=1+\delta_{i}, \forall i$, where $\left|\delta_{i}\right| \leq 1$. In Fig. $4 \mathrm{~b}$, the interconnection of $R(z)$ and $-\delta_{i}$ is shown.

Sufficiency: Since for any number of agents and for any topologies $\left|\delta_{i}\right| \leq 1$ holds, the above spectral radius condition follows from applying the small gain theorem [Skogestad and Postlethwaite, 2005].

Necessity: Define the sets $\mathbf{C}:=\{\delta|\delta \in \mathbb{C},| \delta \mid=1\}$, and $\mathbf{B}:=\{\delta \mid \delta \in \mathbb{R},-1 \leq \delta \leq 1\}$. Let $F(z)$ be a filter (a)

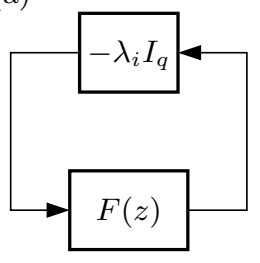

(b)

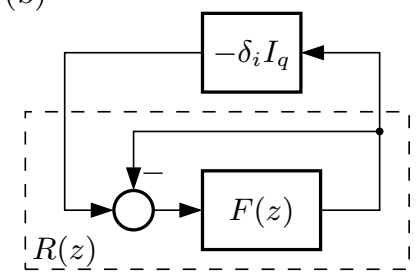

Fig. 4. Feedback interconnections for stability analysis

guaranteeing stability for all topologies. Particularly, the following topologies are stabilized:

- A MAS with $N=2$ agents, and one communication link from agent 1 to agent 2. For this topology $\lambda_{1}=0$ and $\lambda_{2}=1$ and hence $F(z)$ and $R(z)$ are stable.

- A MAS with a cyclic directed communication topology with $N$ agents, where $\mathcal{N}_{i}=\{i+1\}$ for every $i=1, \ldots, N-1 ; \mathcal{N}_{N}=\{1\}$. For this topology $\delta_{i}=-e^{\mathrm{j} \frac{2 \pi i}{N}}, \forall i=1, \ldots, N$, are equidistant points on the unit circle, $\left|\delta_{i}\right|=1, \forall i$. Let $N \rightarrow \infty$. Then the condition for $F(z)$ stabilizing this topology is equivalent to the stability of the interconnection in Fig. 4b for all $\delta \in \mathbf{C}$.

- A MAS with a cyclic undirected communication topology, where $\delta_{i}=-\cos \left(\frac{2 \pi i}{N}\right), \forall i=1, \ldots, N$. Let $N \rightarrow \infty$. Then the condition for $F(z)$ stabilizing this topology is equivalent to the stability of the interconnection in Fig. 4b for all $\delta \in \mathbf{B}$.

Hence $R(z)$ is a stable transfer function and analytic on the unit disk described by $z=e^{\mathrm{j} \omega}$ for all $\omega \in[-\pi, \pi]$. Also the feedback interconnection of $R(z)$ with $-\delta I_{q}$ is stable for all $\delta \in \mathbf{C} \cup \mathbf{B}$. By applying the multi-variable Nyquist stability test [Skogestad and Postlethwaite, 2005] it follows that the Nyquist plot of $\operatorname{det}\left(I_{q}+R\left(e^{\mathrm{j} \omega}\right) \delta\right)$ does not pass through the origin $\forall \omega \in[-\pi, \pi], \forall \delta \in\{\mathbf{C} \cup \mathbf{B}\}$ and also does not encircle the origin.

Rewriting the first condition leads to $\operatorname{det}\left(I_{q}+R\left(e^{\mathrm{j} \omega}\right) \delta\right) \neq$ $0, \forall \omega \in[-\pi, \pi], \forall \delta \in\{\mathbf{C} \cup \mathbf{B}\}$. Dividing by $\delta \neq 0$ leads to $\operatorname{det}\left(I_{q} / \delta+R\left(e^{\mathrm{j} \omega}\right)\right) \neq 0, \quad \forall \omega \in[-\pi, \pi], \forall \delta \in\{\mathbf{C} \cup \mathbf{B} \backslash 0\}$

or, in other words, $R(z)$ does not have complex eigenvalues with modulus 1 , as well as real eigenvalues with modulus greater than $1, \forall z=e^{\mathrm{j} \omega}, \omega \in[-\pi, \pi]$.

Now it will be shown by contradiction that $R\left(e^{\mathrm{j} \omega}\right)$ cannot have eigenvalues with magnitude greater than one for any frequency. Assume that there exist frequencies $\omega_{a}$ and $\omega_{b}$, $0 \leq \omega_{a}<\omega_{b} \leq \pi$, s.t. $\rho\left(R\left(e^{\mathrm{j} \omega_{a}}\right)\right)>1$ and $\rho\left(R\left(e^{\mathrm{j} \omega_{b}}\right)\right)<1$. Then there must exist a frequency $\omega_{x}, \omega_{a}<\omega_{x}<\omega_{b}$, s.t. $\rho\left(R\left(e^{\mathrm{j} \omega_{x}}\right)\right)=1$ and hence there must exist an eigenvalue $\theta_{k}$ of $R\left(e^{\mathrm{j} \omega_{x}}\right)$, s.t. $\left|\theta_{k}\right|=1$. This however contradicts the requirement that there is no eigenvalue of $R\left(e^{\mathrm{j} \omega}\right)$ with magnitude 1. Now assume that $\rho\left(R\left(e^{\mathrm{j} \omega}\right)\right)>1, \forall \omega \in$ $[-\pi, \pi]$. However, since $\lim _{\omega \rightarrow 0} R\left(e^{\mathrm{j} \omega}\right)=R(1) \in \mathbb{R}^{q \times q}$ and since $\operatorname{det}(R(1)) \in \mathbb{R}$, it follows that $R(1)$ has a real eigenvalue with modulus greater than 1 , which contradicts (6).

The above theorem reduces the synthesis problem for a formation with a possibly large number of vehicles to the synthesis of a scalar information filter, which can be performed using standard robust stability analysis/synthesis 
techniques. Furthermore, because in many cases the $q$ channels in each signal can be treated independently, the problem can be reduced to the synthesis of a SISO filter $F_{1}(z)$, and then the filter $F(z)$ can be constructed as $I_{q} \otimes F_{1}(z)$. In this case the spectral radius condition can be replaced by the $\mathcal{H}_{\infty}$ norm of $R(z)$.

In order to find an information flow filter $F(z)$ that not only guarantees stability but also possesses certain dynamic properties, one needs to impose additional performance requirements, e.g., using robust performance synthesis tools. For this purpose construct a generalized plant $G(z)$ as shown in Fig. 5, where $W_{S}(z)$ is a weighting filter expressing sensitivity constraints on the speed of information convergence. Then, in order to design a robust

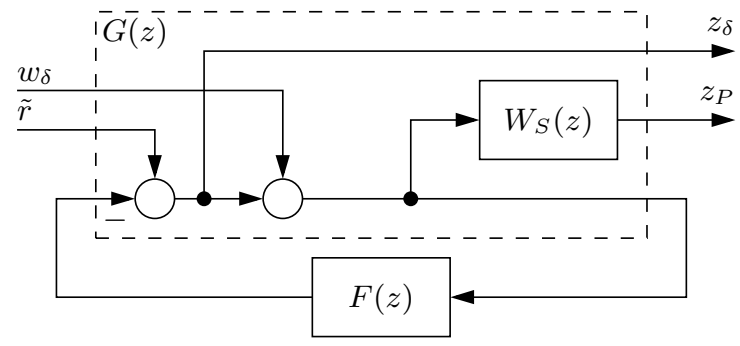

Fig. 5. Interconnection of filter and generalized plant $G(z)$

performance filter one has to find $F(z)$, s.t. the structured singular value $\mu_{\boldsymbol{\Delta}}$ of $T(z)=\mathcal{F}_{L}(G(z), F(z))$ satisfies

$$
\mu_{\boldsymbol{\Delta}}(T(z))<1,
$$

where $\boldsymbol{\Delta}:=\left\{\left[\begin{array}{cc}\delta I_{q} & 0 \\ 0 & \Delta_{P}\end{array}\right]|| \delta \mid \leq 1,\left\|\Delta_{P}\right\| \leq 1\right\}$. Note that the spectral radius condition is a special case of structured uncertainty, i.e., for $\Delta:\{\delta|| \delta \mid \leq 1\}$ it holds $\mu_{\boldsymbol{\Delta}}(R(z))=\max _{\omega \in[-\pi, \pi]} \rho\left(R\left(e^{\mathrm{j} \omega}\right)\right)$. Such a synthesis can be performed by tuning $W_{S}(z)$ using mixed-sensitivity tuning rules and using $\mu$-synthesis techniques (e.g., D-K iterations).

\section{SIMULATION RESULTS}

In this section we illustrate the proposed synthesis of the information flow filter with two example scenarios.

\subsection{Agent Model and Local Controller}

We consider the problem of formation control used in [Fax and Murray, 2004]. Each vehicle is a hovercraft, assumed to move independently in $\mathrm{x}$ - and $\mathrm{y}$-direction and to have identical dynamics in both directions; thus it is possible to reduce the problem to a single-dimensional one (control of the $\mathrm{y}$-position). The vehicle dynamics are described by

$$
P(z)=\frac{y_{i}(z)}{u_{i}(z)}=0.05 \frac{z+1}{(z-1)^{2}} z^{-2},
$$

where a sampling time of $T_{S}=0.1 \mathrm{~s}$ is assumed. Furthermore, we assume that $\phi_{i}=y_{i}$ and that the actuator signals satisfy $\left|u_{i}(t)\right| \leq 5, \forall i, t$.

A local controller $K(z)$ is designed that stabilizes the hovercraft dynamics and is tuned for fast tracking of a step command and fast disturbance rejection. The closedloop response of a single vehicle is shown in Fig. 6 .

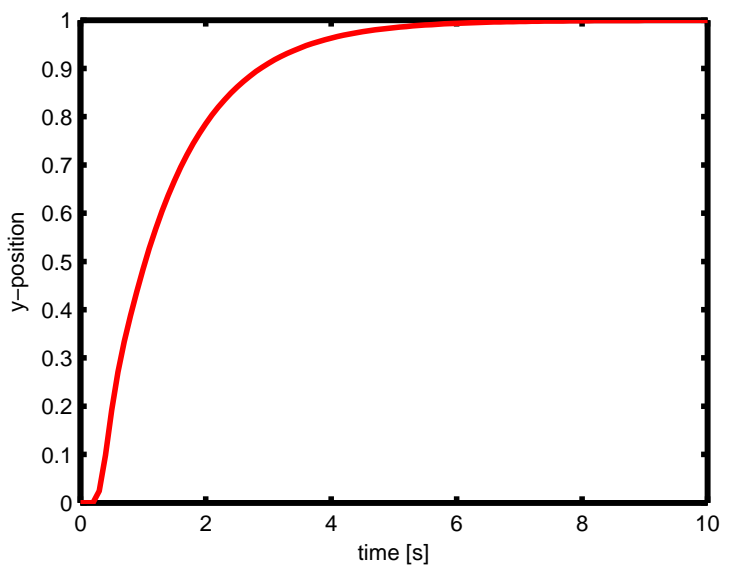

Fig. 6. Response of a single hovercraft to a step reference command

\subsection{Information Flow Filter}

Four different information flow filters are designed. The first one is the heuristically tuned filter $F_{2}(z)$ proposed in [Fax and Murray, 2004]. The other three filters are designed with different shaping filters $W_{S}(z)$ using D$\mathrm{K}$ iterative synthesis with static D-scalings. Filter $F_{f}(z)$ is tuned for fast information convergence, filter $F_{s}(z)$ for slow information convergence but without overshoot, and filter $F_{*}(z)$ is tuned using standard mixed-sensitivity tuning rules. All four filters guarantee the stability of the vehicle formation under arbitrary topology; their transfer functions are:

$$
\begin{aligned}
& F_{2}(z)=0.1875 \frac{z-0.1}{(z-0.25)(z-1)}, \\
& F_{*}(z)=0.28351 \frac{z-0.6784}{(z-0.7)(z-0.9995)}, \\
& F_{f}(z)=0.60895 \frac{z-0.4491}{(z-0.5)(z-0.997)}, \\
& F_{s}(z)=0.13258 \frac{z-0.795}{(z-0.8)(z-0.9999)} .
\end{aligned}
$$

The corresponding filter $R(z)$ is computed as $R(z)=$ $F(z)(1+F(z))^{-1}$; step responses are shown in Fig. 7. The

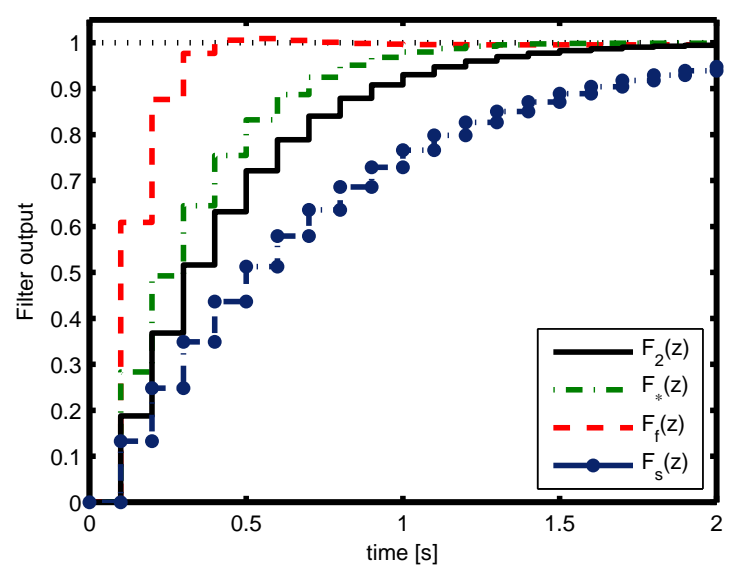

Fig. 7. Step responses of the information filters

requirement that the information filter $F(z)$, and respectively $R(z)$, is strictly proper is satisfied by setting the 
feed-through matrix of the filter to zero during a synthesis using linear matrix inequalities (see, e.g., [D'Oliveira et al., 1999]).

\subsection{Simulation Scenarios}

We consider three example scenarios. In the first two, six hovercrafts are required to attain a desired formation under a known and fixed topology. The two topologies considered are

- (T1) a cyclic communication topology - shown in Fig. 8.a,

- (T2) the communication topology from Figure 2 in [Fax and Murray, 2004] - shown in Fig. 8.b.
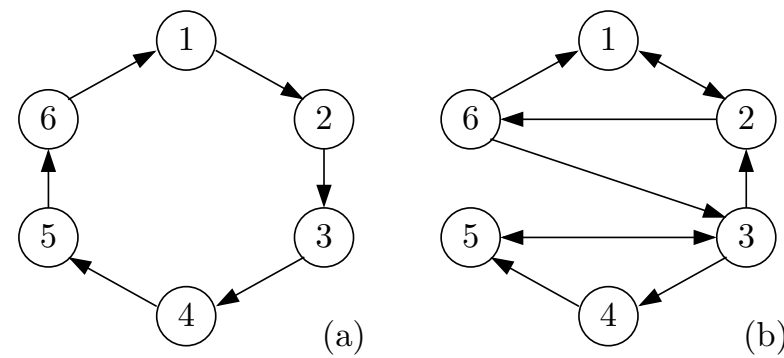

Fig. 8. Example communication topologies

All hovercrafts start at position 0 in y-direction. The formation receives a reference command $r=\left[\begin{array}{lllll}1 & 2 & 3 & 4 & 5\end{array}\right]^{\top}$, which means that the vehicles should position themselves at $y=\psi+1, \psi+2, \ldots$, respectively, for some $\psi \in \mathbb{R}$, since neither topology includes a leader agent.

In the third scenario 10 hovercrafts starting from positions from 1 to 10 are required to rendezvous, with a communication topology that changes randomly every $0.5 \mathrm{~s}$, and with communication delays that vary in a range from 1 to 4 sampling periods. Furthermore, an output step disturbance $d_{y_{4}}$ is acting on agent 4 at $t_{\text {dist }}=5 \mathrm{~s}$. Note that although formation stability with respect to switching communication topologies and time-varying communication delays in Theorem 2 is not proved, simulation results indicate the practical use of the controller design in this setting. This is also true for the case of an output disturbance which affects only one agent and not the whole formation. In this scenario the filter $F_{*}(z)$ is used. Fig. 10 shows the simulation results.

\subsection{Simulation Results}

The simulation results with the four filters for topologies $\mathrm{T} 1$ and $\mathrm{T} 2$ are summarized in Table 1 . The reported times $t_{s 4}$ are the times for which the output of agent 4 settles within a $5 \%$ band of the steady-state value and should serve as an indicator of the settling time of the overall formation. The value $u_{\max }$ is the maximum amplitude of control action in all channels $i$ to a reference step command. The maximum control action to a unit output disturbance is 4.93; it is the same with all filters (due to the common local controller). The formation responses with filters $F_{*}(z)$ and $F_{f}(z)$ and topology T1 are shown in Fig. 9.

Several conclusions can be drawn from the results in Table 1 and Fig. 9. First, by comparing the results of
Table 1. Numerical results

\begin{tabular}{|c||c|c||c|c|}
\hline \multicolumn{1}{|c||}{$\begin{array}{c}\text { Information } \\
\text { filter }\end{array}$} & \multicolumn{4}{c|}{ Topology } \\
$t_{s 4}[\mathrm{~s}]$ & $u_{\max }$ & $t_{s 4}[\mathrm{~s}]$ & $u_{\max }$ \\
\hline \hline$F_{2}(z)$ & 4.8 & 0.89 & 5.8 & 0.72 \\
$F_{*}(z)$ & 4.0 & 1.18 & 5.1 & 0.97 \\
$F_{f}(z)$ & 4.5 & 1.99 & 4.2 & 1.74 \\
$F_{s}(z)$ & 7.3 & 0.61 & 7.6 & 0.49 \\
\hline
\end{tabular}

$F_{2}(z)$ with the systematic synthesis of a robust filter $F_{*}(z)$ the advantage of synthesizing the information flow filter in a systematic way and tuning it using mixed-sensitivity techniques are clearly to be seen. Second, both Table 1 and Fig. 9 show that tuning the information filter for fast convergence might not be advantageous as the actual positions of the vehicles become oscillatory. This can be easily explained based on Fig. 3, where the information filter acts as a prefilter for the local control loop. Hence, if the local controller $K(z)$ is tuned for fast disturbance rejection and a very fast information filter is used, the reference command is directly passed to the local loop and may excite oscillatory modes. Note that this can be avoided by redesigning the local controller and reducing its speed of response, but this could have undesirable effects on disturbance rejection in the local loop. A solution to this problem may be found in the joint design of the information flow filter and the local formation controller.

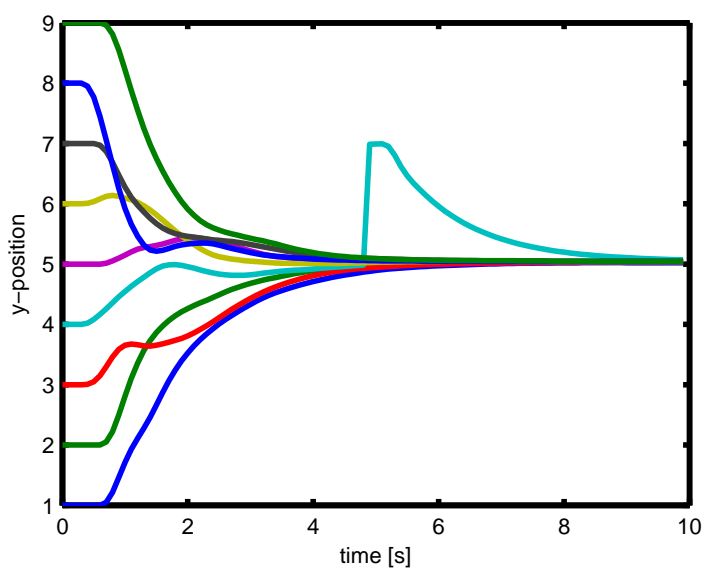

Fig. 10. Formation response with time-varying topology, time-varying communication delays and output disturbance

\section{CONCLUSIONS}

This paper proposes a synthesis procedure for formation control, based on mixed-sensitivity design and a separation theorem. This enables a small gain approach to the synthesis of an information flow filter that improves decentralized controller design. The proposed approach is attractive as it leads to an information flow filter that guarantees stability of a vehicle formation for arbitrary communication topologies and arbitrary communication delays, and can be efficiently synthesized using standard robust control tools. Furthermore, by imposing performance requirements one can tune the filter for a good overall formation performance, as demonstrated by the example scenarios. 
Filter $F_{\star}(z)$

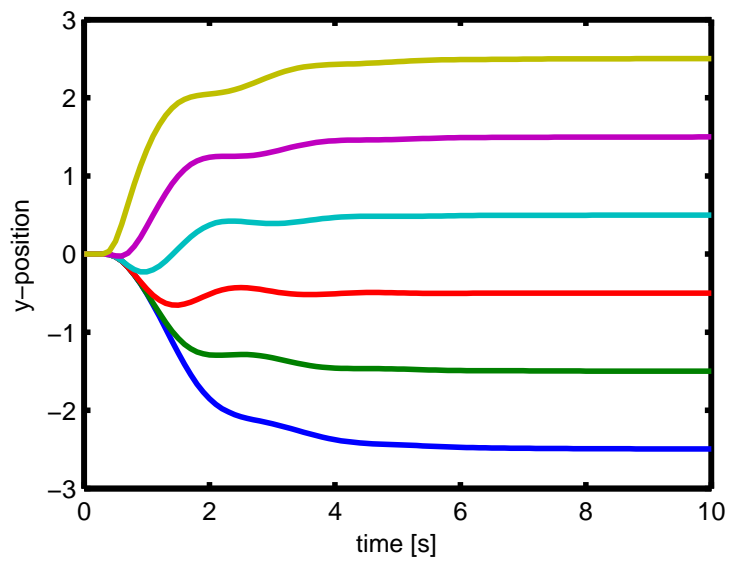

Filter $F_{f}(z)$

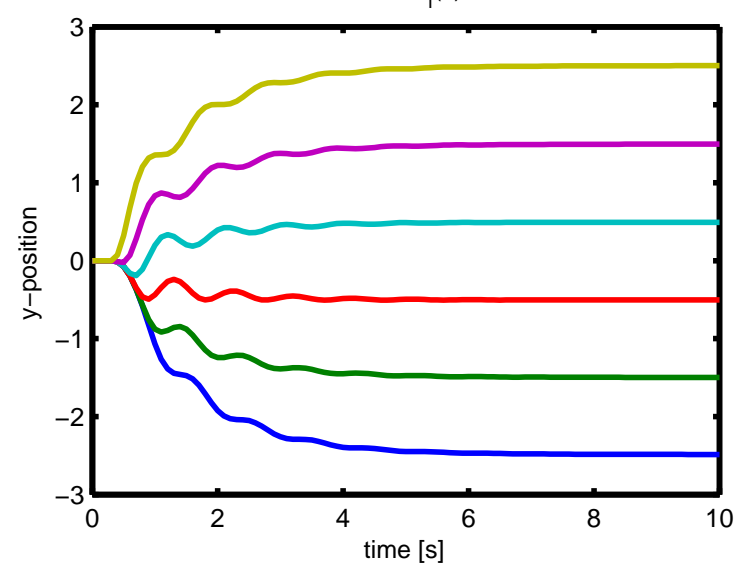

Fig. 9. Formation response with topology $\mathrm{T} 1$ and filter $F_{*}(z)$ (left) and $F_{f}(z)$ (right)

\section{REFERENCES}

Bauso, D., Giarré, L., and Pesenti, R. (2003). Attitude alignment of a team of UAVs under decentralized information structure. In IEEE International Conference on Control Applications, 486-491. Istanbul, Turkey.

Borrelli, F. and Keviczky, T. (2006). Distributed LQR design for dynamically decoupled systems. In Proc. 45th IEEE Conference on Decision and Control, 5639-5644. San Diego, CA, USA.

Bouabdallah, S. and Siegwart, R. (2007). Full control of a quadrotor. In Proc. IEEE/RSJ International Conference on Intelligent Robots and Systems, 153-158. San Diego, CA, USA.

Caughman, J., Lafferriere, G., Veerman, J., and Williams, A. (2005). Decentralized control of vehicle formations. Systems $\&$ Control Letters, 54(9), 899-910.

Chopra, N. and Spong, M. (2006). Advances in Robot Control, chapter Passivity-based Control of Multi-agent Systems, 107-134. Springer Verlag Berlin.

Cortés, J., Martínez, S., and Bullo, F. (2006). Robust rendezvous for mobile autonomous agents via proximity graphs in arbitrary dimensions. IEEE Transactions on Automatic Control, 51(8), 1289-1298.

Cortés, J., Martínez, S., Karatas, T., and Bullo, F. (2004). Coverage control for mobile sensing networks. IEEE Transactions on Robotics and Automation, 20(2), 243255.

Diestel, R. (2005). Graph Theory, volume 173 of Graduate Texts in Mathematics. Springer Verlag.

D'Oliveira, M., Geromel, J., and Bernussou, J. (1999). An LMI optimization approach to multiobjective controller design for discrete-time systems. In Proceedings of the 38th CDC, 3611-1616.

Fax, J.A. and Murray, R. (2004). Information flow and cooperative control of vehicle formations. IEEE Transactions on Automatic Control, 49, 1465-1476.

$\mathrm{Gu}$, D. (2008). A differential game approach to formation control. IEEE Transactions on Control Systems Technology, 16(1), 85-93.

Hussein, I.I. and Stipanović, D.M. (2006). Effective coverage control using dynamic sensor networks. In Proc. 45th IEEE Conference on Decision and Control, 27472752. San Diego, CA, USA.

Kalantar, S. and Zimmer, U.R. (2007). Motion planning for small formations of autonomous vehicles navigating on gradient fields. In Proc. of the International Symposium on Underwater Technology.

Klavins, E. and Murray, R. (2003). Distributed algorithms for cooperative control. IEEE Pervasive Computing, $3(1), 56-65$.

Lin, J., Morse, A., and Anderson, B. (2003). The multiagent rendezvous problem. In Proc. 42nd IEEE Conference on Decision and Control, 1508-1513. Maui, Hawaii, USA.

Liu, Y. and Jia, Y. (2010). $\mathcal{H}_{\infty}$ consensus control of multiagent systems with switching topology - a dynamic output feedback protocol. International Journal of Control, 83(3), 527-537.

Massioni, P. and Verhaegen, M. (2009). Distributed control for identical dynamically coupled systems: A decomposition approach. IEEE Transactions on Automatic Control, 54(1), 124-135.

Olfati-Saber, R. (2006). Flocking for multi-agent dynamic systems: Algorithms and theory. IEEE Transactions on Automatic Control, 51, 401-420.

Ren, W. (2007). Distributed attitude alignment in spacecraft formation flying. International Journal of Adaptive Control and Signal Processing, 21, 95-113.

Ren, W. (2010a). Consensus tracking under directed interaction topologies: Algorithms and experiments. IEEE Transactions on Control Systems Technology, 1(18), 230-237.

Ren, W. (2010b). Distributed cooperative attitude stabilization and tracking for multiple rigid bodies. IEEE Transactions on Control Systems Technology, 2(18), 383-392.

Skogestad, S. and Postlethwaite, I. (2005). Multivariable Feedback Control - Analysis and Design. John Wiley \& Sons, Ltd.

Tanner, H., Jadbabaie, A., and Pappas, G. (2007). Flocking in fixed and switching networks. IEEE Transactions on Automatic Control, 52(5), 863-868.

Unwin, S.C., Shao, M., and Edberg, S.J. (2008). Precision astrometry with a space-based interferometer. In Proc. of SPIE 7013. doi:10.1117/12.790018.

Wang, J. and Elia, N. (2009). Controller synthesis for reusable agents of consensus networks. In Proc. 1st IFAC Workshop on Estimation and Control of Networked Systems, 102-107. Venice, Italy.

Zhou, K., Doyle, J., and Glover, K. (1996). Robust and Optimal Control. Prentice-Hall, N.J., USA. 